\title{
Transcriptional Regulation of Vasopressin Gene: Update in 2015
}

\author{
Yasumasa IWASAKI ${ }^{1, *}$ and Keiichi ITOI $^{2}$ \\ ${ }^{1}$ Health Care Center, and Department of Endocrinology, Metabolism and Nephrology, \\ Kochi University, Kochi 780-8520, Japan \\ ${ }^{2}$ Laboratory of Information Biology, Graduate School of Information Sciences, \\ Tohoku University, Sendai 980-8579, Japan
}

Received July 7, 2015; final version accepted July 26, 2015

\begin{abstract}
Arginine vasopressin (AVP) is expressed in discrete regions of a mammalian brain, and is involved in various physiological functions including the maintenance of body fluid osmolality, regulation of the hypothalamicpituitary-adrenal axis, and formation of the circadian rhythm. Three types of AVP-expressing neurons, among others, have been studied most extensively; these are magnocellular neuroendocrine neurons in the hypothalamic paraventricular and supraoptic nuclei, parvocellular neuroendocrine neurons in the hypothalamic paraventricular nucleus, and neurons in the suprachiasmatic nucleus. Molecular mechanisms, underlying the regulation of AVP gene expression, are different depending on the neuronal type, and different transcription factors play key roles in mediating activation of AVP gene transcription: for example, circadian locomotor output cycles kaput (CLOCK) and brain and muscle aryl hydrocarbon receptor nuclear translocator-like 1 (BMAL1) may be indispensable in AVP gene expression in the suprachiasmatic nucleus. The activator protein 1 (AP1; Fos/Jun) and cyclic adenosine monophosphate response element-binding protein (CREB)-related transcription factors are regarded as major transcription factors in the parvocellular and magnocellular hypothalamic neurons, respectively. According to recent studies, CREB3-like protein 1 (CREB3L1), a transcription factor of the CREB/activating transcription factor family, may mediate the osmolality-dependent AVP gene transcription in the magnocellular neurons.
\end{abstract}

KEYWORDS: arginine vasopressin, osmoregulation, hypothalamus, transcription

\section{Introduction}

Arginine vasopressin (AVP) is a posterior pituitary hormone, synthesized in the paraventricular and supraoptic nuclei of the hypothalamus and plays a key role in maintaining the osmolality of body fluid. AVP is a nonapeptide, and its chemical structure was disclosed in the mid-twentieth century [1]. The amino acid sequence of AVP is Cys-Tyr-PheGln-Asn-Cys-Pro-Arg-Gly, with the cysteine residues forming a disulfide bond. Arginine residue is present in the side chain of the vasopressin molecule in all mammalian species except for the pig, in which it is substituted by lysine (lysine vasopressin). AVP is one of the neuropeptides that has been studied most extensively probably because it is indispensable for the survival of an organism. Later findings that AVP is present in discrete neurons in the hypothalamus $[2,3]$ contributed greatly to the conceptual framework of 'neuroendocrinology'.

In the brain, AVP is expressed most conspicuously in the magnocellular neuroendocrine neurons of hypothalamic paraventricular nucleus (PVN), and supraoptic nucleus (SON), which take part in water and electrolyte homeostasis, and neurons of the suprachiasmatic nucleus (SCN), which are involved in circadian rhythm formation [4]. AVP is also expressed in the parvocellular neuroendocrine neurons in the PVN which is responsible for the regulation of the hypothalamic-pituitary-adrenal (HPA) axis [5]. In contrast to the magnocellular AVP neurons, AVP is not expressed prominently in basal conditions (unstressed states with normally functioning adrenals) in parvocellular neurons, but its expression is greatly enhanced in conditions such as glucocorticoid (GC) deficiency [6,7].

Although the human genome has a single AVP gene with no alternative promoters, the mechanism of its transcriptional regulation is different across the neuronal populations, and this may be one of the underlying mechanisms through which the AVP molecule plays distinct physiological roles in different brain regions. In this brief review, we summarize the current understandings of how AVP gene expression is regulated differentially in multiple neuronal types, from the viewpoint of transcription factors.

\section{AVP Gene Expression in Parvocellular Neurons}

The corticotropin-releasing hormone (CRH)-expressing neurons belong to the parvocellular neuroendocrine neurons in the PVN, and a proportion of the CRH neurons possess a capability of co-expressing AVP. In the parvocellular PVN, 
AVP expression becomes most conspicuous in GC deficient states as was described above, and AVP-immunoreactivity increases markedly following bilateral adrenalectomy in the rat $[6,7]$. CRH and AVP are co-localized in the same secretory granule at the nerve terminals in the median eminence [8], and they exert a synergistic effect on the secretion of adrenocorticotropin from the anterior pituitary [9]. Therefore, both CRH and AVP are regarded as physiological secretagogues for adrenocorticotropin. Besides GC deficiency, a variety of stressors is known to activate AVP gene transcription in the parvocellular CRH neurons [10]. AVP expression is suppressed very potently by circulating GCs [11], which is probably the reason why AVP expression is not conspicuous in parvocellular neurons under normal circadian rhythm of plasma GC concentration.

Various kinds of stressors trigger an induction of immediate early genes of fos and jun families (and their protein products, Fos and Jun) in the parvocellular division of the PVN [12]. We have examined the involvement of Fos/Jun in the transcriptional regulation of AVP gene in vitro, and found that these inducible transcription factors indeed activate AVP gene expression via a specific AP1 binding site [13]. More recently, we also found that FosB/ $\Delta$ FosB, one of the Fos family members, is expressed in parvocellular neurons of the rat PVN, and its expression is enhanced markedly by bilateral adrenalectomy [14]. Furthermore, GC (dexamethasone) exerted a negative effect on FosB expression both in vitro and in vivo [14]. Since $F o s B / \Delta F o s B$ has an exceptionally long intracellular half-life [15], induction of FosB/ $\Delta$ FosB may underscore the sustained activation of the HPA-axis during hypoadrenocorticism. Interestingly, overexpression of the FosB or $c$-jun gene upregulates CRH mRNA levels in a homologous cell line, 4B, and in contrast, downregulation of FosB or $c$-jun gene suppressed the CRH gene expression induced by the protein-kinase A, proteinkinase $\mathrm{C}$, or intracellular calcium-dependent signal transduction pathway [16]. These findings suggest that FosB, together with c-Jun, may mediate the CRH gene expression in the hypothalamus. It is not clear yet whether the AVP gene expression, in the parvocellular neurons, is also regulated by FosB $/ \Delta$ FosB. Nor is it clear whether the ligand (GC)-bound GRs interact with the heterodimer of FosB $/ \Delta$ FosB and a Jun family protein and repress the latter, as was demonstrated for the interaction between Fos/Jun and GR [17].

\section{AVP Gene Expression in Magnocellular Neurons}

Magnocellular neurons in the SON and PVN comprise AVP- and oxytocin (OXT)-producing neurons, and AVP and OXT are not co-localized in the same neuron; thus AVP- and OXT-containing neurons constitute different subpopulations of magnocellular neurons [2]. AVP and OXT are transported to the posterior pituitary gland together with their carrier proteins, neurophysin II and I, respectively, and released into the systemic circulation. The osmolality of body fluid is regulated strictly by AVP, and conversely, synthesis and secretion of AVP are regulated by plasma osmolality. Two major issues remain unresolved: 1) where in the brain the osmoreceptor(s) is located, what the chemical structure of osmoreceptors is, and how plasma osmolality changes are perceived by the osmoreceptors, and, 2) whether or not the magnocellular vasopressinergic neurons per se are osmosensitive.

Outside the hypothalamus, there are three brain regions which have been implicated in sensing plasma osmolality; these are the subfornical organ (SFO), the median preoptic nucleus, and the organum vasculosum lamina terminalis (OVLT) [18]. Although some neurons in the SFO and OVLT are intrinsically osmosensitive, there has been no direct evidence that the cells in these regions contribute to the osmotic control of neurohypophysial hormone release and/or triggering drinking behaviors. Very recently, Oka and colleagues identified two distinct neural populations in the SFO that trigger or suppress thirst by an optogenetic means, revealing an innate brain circuit that can turn an animal's waterdrinking behavior on and off [19].

Members of the transient receptor potential vanilloid (TRPV) family channels became strong candidates for the molecular detection of osmotic stimuli [20]. TRPV1 and TRPV4 (vanilloid receptor-related osmotically activated channel; VR-OAC), among other TRPV channels, were proposed to be involved in hypertonicity sensing [21]. Ciura and colleagues reported that the neurons in the OVLT, where the primary osmosensory neurons reside, hypertonic stimuli activated a class of non-selective cation channels [21]. OVLT neurons isolated from animals lacking expression of Trpvl failed to show significant increase in conductance in response to hypertonic stimulation, but those from animals lacking $\operatorname{Trpv} 4$ generated significant increase in conductance, and these changes were equivalent to those observed in wild-type OVLT neurons. From these results, it was suggested that TRPV1, but not TRPV4, may be involved in the hypertonicity sensing in OVLT [21]. However, Kinsman and colleagues reported more recently that single or double knockout of Trpv1/Trpv4 did not cause a phenotype of impaired water metabolism, so the physiological significance of these channels is still elusive [22].

Nax, a subclass of sodium (Na) channels, has also been proposed as an osmosensory molecule in the circumventricular organs by Noda and colleagues [23]. It is a sodium concentration sensitive but not voltage sensitive channel and distributed in limited brain regions including the SFO, OVLT, median eminence, and posterior pituitary [23]. Hiyama and colleagues reported a human case report, in which autoantibody against Nax was produced in a patient, and as a result, osmotic regulation of AVP secretion was profoundly impaired [24]. The primary targets of the autoantibodies involved in the development of hypernatremia are most likely Nax-positive cells within the circumventricular organs, because autoantibodies present in the patient's serum bound to the SFO and OVLT of the wild-type mice, but not those of the Nax-KO mice [24]. This case report suggests a permissive role of Nax in the 
sensation of osmolality in humans.

The AVP gene transcription is regulated rapidly in response to constantly changing plasma osmolality [25]. Therefore, signal transduction pathways within the vasopressinergic neuron must readily be activated by the input signals conveyed from the sites where the osmoreceptors reside and trigger phosphorylation of cyclic adenosine monophosphate (cAMP)/protein kinase $\mathrm{A}$ and/or calcium/calmodulin-dependent protein kinases, and then the transcription factor CREB or related factor(s) [26, 27].

It has long been a subject of dispute: are the magnocellular vasopressinergic neurons per se are osmosensitive? There was an argument that changes in plasma osmolality may not be sensed by the magnocellular neurons in the SON and PVN because of the presence of blood-brain barrier. Oilet and Bourque demonstrated the presence of cationic channels in isolated magnocellular neuroendocrine cells which could be reversibly modulated by changes in pipette pressure, or by modifying the osmolality of the solution surrounding the cell [28]. They proposed these channels as possible mechanotransducers for osmoreception [18,28].

Recently, Greenwood and colleagues reported the implication of cyclic adenosine monophosphate (cAMP)-response element-binding protein (CREB) 3-like protein 1 (CREB3L1), a novel endoplasmic reticulum (ER) stress transducer and member of the CREB/activating transcription factor family, as a transcriptional mediator of the ER stress response in the osmotically stimulated SON [29]. CREB3L1 was activated predominantly in vasopressinergic neurons in response to hyperosmotic stress [30]. Furthermore, CREB3L1 binding sites were identified in the rat AVP 5'-promoter region by AVP promoter deletion constructs using heterologous HEK293 kidney cell line, and direct binding of CREB3L1 was shown by chromatin immunoprecipitation both in vitro and in the SON [30]. We have also examined the effects of a variety of ER stress-related transcription factors on AVP gene transcription using homologous neuronal cell line, BE(2)M17, which endogenously expresses AVP mRNA. We found that CREB3L1 stimulates dramatically the $5^{\prime}$-promoter activity of the rat AVP gene ( $>15$-fold) (Fig. 1). However, the intracellular mechanism(s) through which CREB3L1 is activated by hypertonicity remains to be elucidated. In addition, it requires to be examined whether CREB3L1 mediates the neural input-mediated AVP gene transcription, besides the osomoreceptor-mediated mechanism within the AVP neurons.

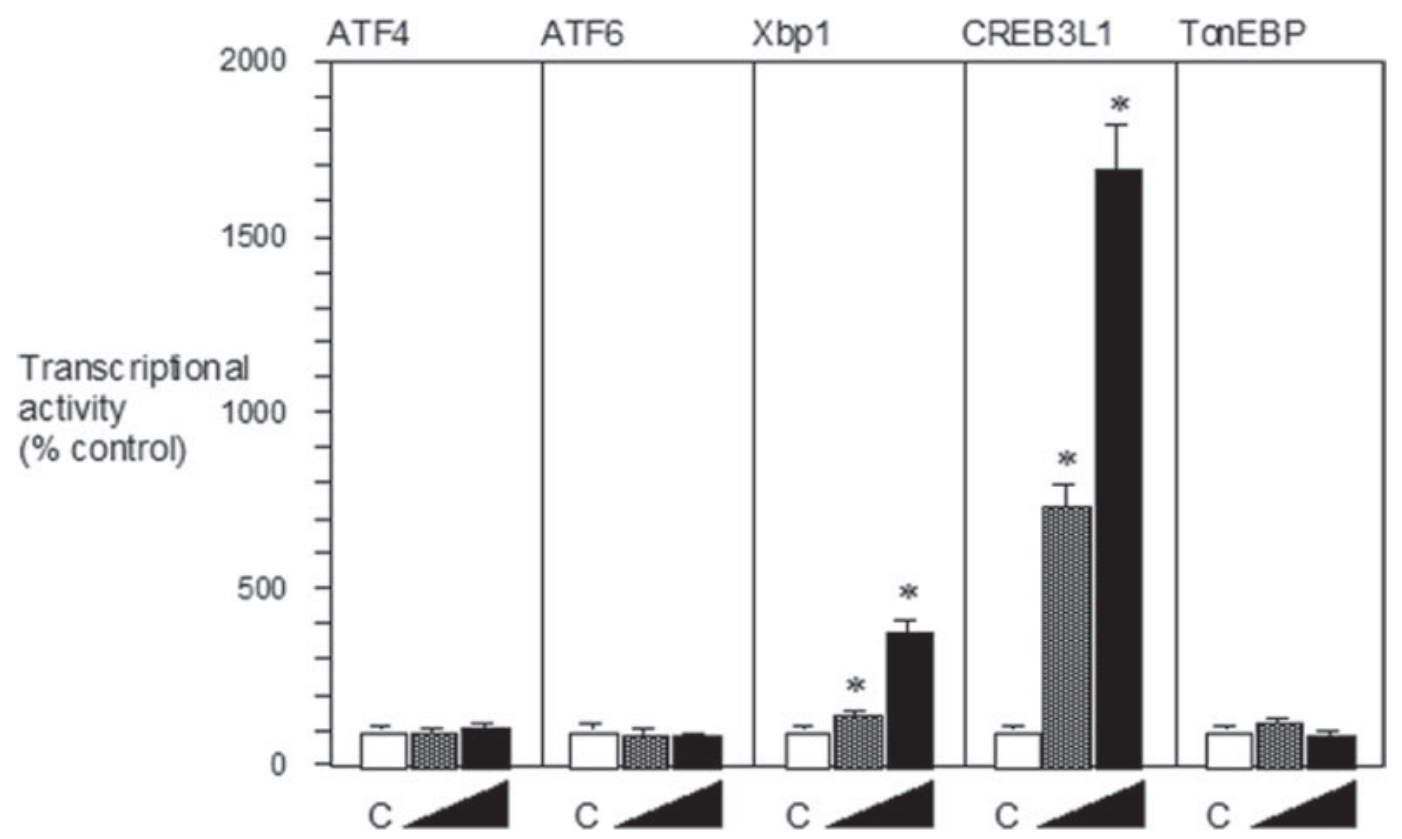

Fig. 1. Effects of the overexpression of ER stress-related and osmosensitive transcription factors on the transcriptional activity of the rat AVP gene

A plasmid containing approximately $0.5 \mathrm{~kb}$ of the rat AVP promoter regions flanking the luciferase construct gene was transfected to the BE(2)M17 neuronal cell line. Another plasmid containing either of the ER stress-related or osmosensitive transcription factors was co-transfected, and the reporter assay was carried out. The ER stress-related transcription factors include activating transcription factor 4 (ATF4), ATF6, a UPR-specific splice variant of X-box binding protein 1 (Xbp1s), and CREB3L1. The tonicity response element binding protein (TonEBP) was used as an osmosensitive transcription factor.

\section{AVP Gene Expression in the SCN}

AVP mRNA and AVP peptide are expressed prominently in the SCN [31]. Since AVP gene promoter contains the E-box (CACGTG), the gene has been postulated to be under the control of circadian clock genes (Clock/Bmall), and 
indeed, this hypothesis was demonstrated to be true both in vivo and in vitro [32,33]. Interestingly, in the SCN, AVP neuron-specific disruption of the core circadian transcription factor, BMAL1, dramatically decreases AVP gene expression (Dr. Sakutai T, Kanazawa University, personal communication), along with impairment in oscillation network within the SCN [34]. These results suggest that the expression levels of AVP gene are controlled by the core circadian transcription factors CLOCK/BMAL1 in the SCN, and AVP peptide may also play an important role in the circadian regulatory mechanism within the SCN. Furthermore, the AVP V1a receptor subtype-knockout mice showed abnormalities in the circadian rhythm of locomotor activity, suggesting that the action of AVP in the circadian behavioral pattern may at least partly be mediated through the V1a receptor [35].

\section{Summary}

The promoter of AVP gene is regulated differentially in the three distinct types of neurons in the hypothalamus; magnocellular and parvocellular neurons of the SON and/or PVN, and neurons of the SCN. CREB3L1, AP1 (Fos/Jun), and CLOCK/BMAL1 are recognized as the major transcription factors in each neuronal population (Fig. 2). Considering that the osmoregulation is crucial for survival, it may be plausible that an organism is equipped with dual functional mechanisms of osmoregulation: the intrinsic osmotic sensing mechanism within the AVP neuron, and the mechanism mediated through the extrinsic inputs which derive from the osmoreceptor-expressing neurons outside the PVN and SON. Multiple transcription factors including CREB and CREB3L1 may mediate the regulation of AVP gene expression redundantly, thus forming a transcriptional regulatory network in magnocellular neuroendocrine neurons.

\begin{tabular}{|c|c|c|}
\hline 1000 & AAGATCAAA AGI T CAATGCCAG CTTTCIGCTA TGTAGTAAGGTCAAGGTC & \\
\hline-950 & AGCCIGGAC TAAACGACT GCCT TAGAAACAAG CAAAT TACIT ACCGTCTA & \\
\hline-900 & AAGICAGGA ACTACACTIGCTT TCTCAGACTG TGICT GICT GTCTGGGGC & \\
\hline-850 & TCCTCCCAT TTCCTCTCCT AAC AACATCCACT TCCACTCCIGCCTTAGAT & \\
\hline-800 & CIGAGATAG TACCAGCCI CAGG GCATGGGGIC TCCCCATAGCTTITCCTC & \\
\hline-750 & TGCAGTACT GIGGGCTCACCTA GGACT GITIC CGAACTATATCCTACCCT & \\
\hline-700 & AGCICTCTA CCCTAGAAGGCCT GAAACTCACA GAAAT TCTCCTGCCTCTG & \\
\hline-650 & CTTICCAAT GGCT GGGGT T AAA AGCAT GT GICACAACTGTCCTTTT TATT & \\
\hline-600 & CTTTTAATA TCGAGACAGGGTC TCACCAAGTI GCCCCAAGACGCCAGCCA & \\
\hline-550 & CACCTGGGA CAGGGCAGGCCTI TGGCTCTATG TTCAGTCTIGACTCCATG & \\
\hline-500 & ACTGIGGCC GCTAGCCCAT GAG GCT GCGCGTG GGAATTTCCTTCTGAAAG & \\
\hline-450 & CTCACCTGG TAT CGATGCT TCC TCT TATCCTA CACCACA.ACTAACAAACC & \\
\hline-400 & TGCCCCACC TCCIGGTCCTGAC CCTGCTGCAGACCTGCTAGICCTIGGIG & \\
\hline-350 & AATGAGACC TGGGGACCCCTCT AGTCTGT TGA GAGCTGCTGAAATGCTCA & \\
\hline-300 & ACTATGATT TCCAGGTGACCCT CAAGICGGCT CACCT CCCIGATTGCACA & \\
\hline-250 & GCACCAATCACT GIGGCGGTGG TCCCGTCACACGGT GGCCAGTGACAGC & CAAT bOX CRE1 \\
\hline-200 & CTGATGGCT GGCICCCCICCTC CACCACCCIC AGCAITGACA GGCECAC & $G-b o x$ \\
\hline-150 & 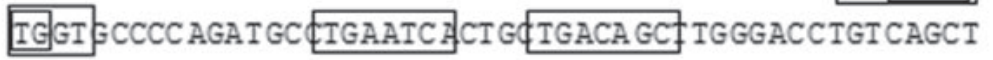 & CRE2 \\
\hline-100 & GTGGGCTCCTGGGGAGCCACTGGGGAGGGGGI TAGCAGCCACGCTGTCGC & \\
\hline-50 & CI CCTAGCC AACACCTGCAGZCATAAATAGACAGCCCAGCCCGCTCAGGC & TATA box \\
\hline+1 & AGCAGAGCA GAGCTGCACGCAGTGCCCACCTa tg & \\
\hline
\end{tabular}

Fig. 2. Nucleotide sequences of the rat AVP gene promoter

The $0.25 \mathrm{~kb}$ of the proximal region (especially between -250 and $-120 \mathrm{bp}$ from the transcription start site; +1 denotes the transcription start site) contains most of the important binding sites (boxed). Human AVP gene promoter has almost identical nucleotide sequences and binding sites (data not shown).

\section{Acknowledgments}

We thank Prof. Kazutoshi Mori (Kyoto University) and Dr. Hiroaki Okuda (Nara Medical University) for Xbp1s and CREB3L1 expression plasmids, respectively. 


\section{REFERENCES}

[1] Popenoe EA, DuVigneaud V (1954) A partial sequence of amino acids in performic acid-oxidized vasopressin. J Biol Chem 206:353-360.

[2] Vandesande F, Dierickx K (1975) Identification of the vasopressin producing and of the oxytocin producing neurons in the hypothalamic magnocellular neurosecretory system of the rat. Cell Tissue Res 164:153-162.

[3] Swaab DF, Pool CW, Nijveldt F (1975) Immunofluorescence of vasopressin and oxytocin in the rat hypothalamoneurohypophypopseal system. J Neural Transm 36:195-215.

[4] Ibata Y, Okamura H, Tanaka M, Tamada Y, Hayashi S, Iijima N, Matsuda T, Munekawa K, Takamatsu T, Hisa Y, Shigeyoshi Y, Amaya F (1999) Functional morphology of the suprachiasmatic nucleus. Front Neuroendocrinol 20:241-268.

[5] Itoi K, Jiang YQ, Iwasaki Y, Watson SJ (2004) Regulatory mechanisms of corticotropin-releasing hormone and vasopressin gene expression in the hypothalamus. J Neuroendocrinol 16:348-355.

[6] Kiss JZ, Mezey E, Skirboll L (1984) Corticotropin-releasing factor-immunoreactive neurons of the paraventricular nucleus become vasopressin positive after adrenalectomy. Proc Natl Acad Sci USA 81:1854-1858.

[7] Itoi K, Mouri T, Takahashi K, Murakami O, Imai Y, Sasaki S, Yoshinaga K, Sasano N (1987) Suppression by glucocorticoid of the immunoreactivity of corticotropin-releasing factor and vasopressin in the paraventricular nucleus of rat hypothalamus. Neurosci Lett 73:231-236.

[8] Whitnall MH, Mezey E, Gainer H (1985) Co-localization of corticotropin-releasing factor and vasopressin in median eminence neurosecretory vesicles. Nature 317:248-250.

[9] Rivier C, Rivier J, Mormede P, Vale W (1984) Studies of the nature of the interaction between vasopressin and corticotropinreleasing factor on adrenocorticotropin release in the rat. Endocrinology 115:882-886.

[10] Aguilera G, Rabadan-Diehl C (2000) Vasopressinergic regulation of the hypothalamic-pituitary-adrenal axis: implications for stress adaptation. Regul Pept 96:23-29.

[11] Helmreich DL, Itoi K, Lopez-Figueroa MO, Akil H, Watson SJ (2001) Norepinephrine-induced CRH and AVP gene transcription within the hypothalamus: differential regulation by corticosterone. Mol Brain Res 88:62-73.

[12] Kovács KJ (1998) Functional neuroanatomy of the parvocellular vasopressinergic system: transcriptional responses to stress and glucocorticoid feedback. Prog Brain Res 119:31-43.

[13] Yoshida M, Iwasaki Y, Asai M, Takayasu S, Taguchi T, Itoi K, Hashimoto K, Oiso Y (2006) Identification of a functional AP1 element in the rat vasopressin gene promoter. Endocrinology 147:2850-2863.

[14] Das G, Uchida K, Kageyama K, Iwasaki Y, Suda T, Itoi K (2009) Glucocorticoid dependency of surgical stress-induced FosB/ $\triangle$ FosB expression in the paraventricular and supraoptic nuclei of the rat hypothalamus. J Neuroendocrinol 21:822-831.

[15] Carle TL, Ohnishi YN, Ohnishi YH, Alibhai IN, Wilkinson MB, Kumar A, Nestler, EJ (2007) Proteasome-dependent and -independent mechanisms for FosB destabilization: identification of FosB degron domains and implications for DeltaFosB stability. Eur J Neurosci 25:3009-3019.

[16] Kageyama K, Itoi K, Iwasaki Y, Niioka K, Watanuki Y, Yamagata S, Nakada Y, Das G, Suda T, Daimon M (2014) Stimulation of corticotropin-releasing factor gene expression by FosB in rat hypothalamic 4B cells. Peptides 51:59-64.

[17] Karin M, Chang L (2001) AP-1-glucocorticoid receptor crosstalk taken to a higher level. J Endocrinol 169:447-451.

[18] Bourque CW, Oliet SH (1997) Osmoreceptors in the central nervous system. Annu Rev Physiol 59:601-619.

[19] Oka Y, Ye M, Zuker CS (2015) Thirst driving and suppressing signals encoded by distinct neural populations in the brain. Nature 520:349-352.

[20] Liedtke W, Choe Y, Martí-Renom MA, Bell AM, Denis CS, Sali A, Hudspeth AJ, Friedman JM, Heller S (2000) Vanilloid receptor-related osmotically activated channel (VR-OAC), a candidate vertebrate osmoreceptor. Cell 103:525-535.

[21] Ciura S, Liedtke W, Bourque CW (2011) Hypertonicity sensing in organum vasculosum lamina terminalis neurons: a mechanical process involving TRPV1 but not TRPV4. J Neurosci 31:14669-14676.

[22] Kinsman B, Cowles J, Lay J, Simmonds SS, Browning KN, Stocker SD (2014) Osmoregulatory thirst in mice lacking the transient receptor potential vanilloid type 1 (TRPV1) and/or type 4 (TRPV4) receptor. Am J Physiol 307:R1092-R1100.

[23] Noda M, Hiyama TY. The Nax Channel (2015) What It Is and What It Does. Neuroscientist 21:399-412.

[24] Hiyama TY, Matsuda S, Fujikawa A, Matsumoto M, Watanabe E, Kajiwara H, Niimura F, Noda M (2010) Autoimmunity to the sodium-level sensor in the brain causes essential hypernatremia. Neuron 66:508-522.

[25] Arima H, Kondo K, Kakiya S, Nagasaki H, Yokoi H, Yambe Y, Murase T, Iwasaki Y, Oiso Y (1999) Rapid and sensitive vasopressin heteronuclear RNA responses to changes in plasma osmolality. J Neuroendocrinol 11:337-341.

[26] Pardy K, Adan RA, Carter DA, Seah V, Burbach JP, Murphy D (1992) The identification of a cis-acting element involved in cyclic 3',5'-adenosine monophosphate regulation of bovine vasopressin gene expression. J Biol Chem 267:21746-21752.

[27] Iwasaki Y, Oiso Y, Saito H, Majzoub JA (1997) Positive and negative regulation of the rat vasopressin gene promoter. Endocrinology 138:5266-5274.

[28] Oliet SH, Bourque CW (1993) Mechanosensitive channels transduce osmosensitivity in supraoptic neurons. Nature 364:341-343.

[29] Greenwood M, Greenwood MP, Paton JF, Murphy D (2015) Transcription factor CREB3L1 regulates endoplasmic reticulum stress response genes in the osmotically challenged rat hypothalamus. PLoS One 10:e0124956.

[30] Greenwood M, Bordieri L, Greenwood MP, Rosso Melo M, Colombari DS, Colombari E, Paton JF, Murphy D (2014) Transcription factor CREB3L1 regulates vasopressin gene expression in the rat hypothalamus. J Neurosci 34:3810-3820.

[31] Robinson BG, Frim DM, Schwartz WJ, Majzoub JA (1988) Vasopressin mRNA in the suprachiasmatic nuclei: daily regulation of polyadenylate tail length. Science 241:342-344.

[32] Ghorbel MT, Coulson JM, Murphy D (2003) Cross-talk between hypoxic and circadian pathways: cooperative roles for hypoxia-inducible factor 1alpha and CLOCK in transcriptional activation of the vasopressin gene. Mol Cell Neurosci 
22:396-404.

[33] Maruyama T, Ohbuchi T, Fujihara H, Shibata M, Mori K, Murphy D, Dayanithi G, Ueta Y (2010) Diurnal changes of arginine vasopressin-enhanced green fluorescent protein fusion transgene expression in the rat suprachiasmatic nucleus. Peptides 31:2089-2093.

[34] Mieda M, Ono D, Hasegawa E, Okamoto H, Honma K, Honma S, Sakurai T (2015) Cellular clocks in AVP neurons of the SCN are critical for interneuronal coupling regulating circadian behavior rhythm. Neuron 85:1103-1116.

[35] Li JD, Burton KJ, Zhang C, Hu SB, Zhou QY (2009) Vasopressin receptor V1a regulates circadian rhythms of locomotor activity and expression of clock-controlled genes in the suprachiasmatic nuclei. Am J Physiol 296:R824-R830. 